\title{
Development of a gel monolithic column polydimethylsiloxane microfluidic device for rapid electrophoresis separation
}

\author{
Hu-Lie Zeng, Hai-Fang Li, Xu Wang, Jin-Ming Lin* \\ State Key Laboratory of Environmental Chemistry and Ecotoxicology, Research Center for Eco-Environmental Sciences, \\ Chinese Academy of Science, P.O. Box 2871, Beijing 100085, China
}

Received 13 July 2005; received in revised form 22 September 2005; accepted 22 September 2005

Available online 20 October 2005

\begin{abstract}
A $\beta$-cyclodextrin ( $\beta$-CD)-bonded gel monolithic column polydimethylsiloxane (PDMS) microfluidic device was developed in a simple and feasible way. Before preparation of gel monolithic column in PDMS microchannel, PDMS surface was activated by UV light to create silanol groups, which is an active molecule to covalently bond 3-(trimethoxysilyl)-propyl methacrylate (Bind-Silane) and seal microfluidic device. By the way, Bind-Silane is a bifunctional molecule to link polyacrylamide (PAA) gel and inner wall of PDMS microchannel covalently. Allyl- $\beta$-CD was used not only as a multifunctional crosslinker in PAA gel to control the size of the pores, but also as a chiral selector for the enantioseparation. The stability, transferring heat and optical characteristic of the microfluidic device were examined. The separation capability of the gel monolithic column was confirmed by the successful separation of fluorescein isothiocyanate (FITC)-labeled arginine (Arg), glutamine acid (Glu), tryptophan (Try), cysteine (Cysteine) and phenylalanine (Phe) in the PDMS microfluidic device less than $100 \mathrm{~s}$ at $36 \mathrm{~mm}$ effective separation length. A maximum of $2.06 \times 10^{5}$ theoretical plates was obtained by the potential strength of $490 \mathrm{~V} / \mathrm{cm}$. A pair of FITC-labeled dansyl-D,L-threonine (Dns-Thr) was separated absolutely.
\end{abstract}

(C) 2005 Elsevier B.V. All rights reserved.

Keywords: PDMS microfluidic device; Monolithic column; Chiral separation; FITC-labeled amino acid

\section{Introduction}

In the past years, many kinds of material have been used as raw material for fabricating microfluidic devices, such as silicone [1], glass [2], quartz and polymers [3-5]. Among these materials, polymers have been focused on greatly because of universal raw materials, low cost, accessible to large-scale manufacture and other especial peculiarities. In terms of the polymers, polydimethylsiloxane (PDMS) is an important material for fabrication of microfluidic devices. It is low cost and well durability with excellent optical speciality [6,7]. Certainly, the development of the technology of the seal irreversibly or reversibly of PDMS microfluidic device with PDMS, polymethylacrylate (PMMA) and glass is also critical $[8,9]$, which has been applied to fabricate various microfluidic devices widely. However, Microsystems fabricated from PDMS exhibit a number of

\footnotetext{
* Corresponding author. Tel.: +86 1062841953 ; fax: +86 1062841953 .

E-mail address: jmlin@ mail.rcees.ac.cn (J.-M. Lin).
}

weaknesses when they are considered employing electrophoresis [9-12]. One of the disadvantages is the extreme hydrophobicity of PDMS making the microdevices difficultly to fill with aqueous solutions and ensure stable and easily controlled electroosmotic flow (EOF).

Approaches to overcome the shortcoming of PDMS for electrophoretic applications are performed including chemical and physical methods. The aim of both means is to stabilize the surface speciality of PDMS and tailor separating function of PDMS microchannel. The physical methods include physical adsorbing stationary phases and dynamic coating on PDMS surface [12]. Many polymers, such as polyglycol, polyacrylamide (PAA) and cetyltrimethyl ammonium bromide (CTAB) have been widely applied as hydrophilic functional materials for PDMS microchannel to bring down its surface hydrophobicity. Some ways about changing the surface of PDMS substrate through the chemical means have been also carried out $[13,14]$. For instance, Hu et al. [13] had proposed the technique about surface-directed grafting polymerization in PDMS-based microfluidic device. In this case, the hydrophobicity of PDMS-based microchannel 
has been changed through the surface grafted with acrylic acid, poly(ethylene glycol) monomethoxyl acrylate and poly(ethylene glycol) diacrylate. Subsequently, Slentz et al. [14] had performed $\mathrm{Ce}(\mathrm{IV})$ catalyzed polymerization on the PDMS microchannnel inner wall to prepare a reproducible and stable coating to separate peptide mixtures. On this view, bonding stationary phase on inner wall of microchannel is a powerful mean to change the surface peculiarity of PDMS to improve its hydrophilicity. Therefore, changing PDMS surface hydrophobicity through chemical ways seems to obtain farther steady surface property for electrophoresis.

Furthermore, the approach to prepare monolithic column in situ in capillary or microchannel has been a potential mode to avoid an expensive synthesis of beads and the cumbersome packing [15]. Generally, monolithic column as a separation media for electrophoresis or electrochromatography is superior to continuous beds and packed bed. Because homogeneous gel of monolithic column does not consist of particles; it can give much narrower zones [16]. In recent years, much study correlative to monolithic column has been widely performed. The group of Kato [17] had demonstrated the highly efficiency of monolithic column for electrochromatography in the separation of amino acids (AAs). Noticeably, Tian et al. [18,19] have done much work in the exploring the application of gel monolithic column in life sciences for capillary or microchip electrochromatography. Based on the widely adoption of gel monolithic column in contemporary analytical science, many complicated DNA and protein have been well separated in gel monolithic column for electrochromatograohy [17-19]. On the other hand, the chiral separation of gel monolithic column for electrochromatography has been focused on recently. PAA gel has been used to prepare monolithic column for electrochromatography because of its well stability and reproducibility. With the demand of analyzing enantiomers, the chiral separation and PAA gel monolithic column have been integrated to explore novel chiral separation methods. The groups of Vébvári [20] and Koide [21] prepared $\beta-\mathrm{CD}$ bonded PAA gel monolithic column for electrochromatograohy, which was used to separate the enantiomers of amino acids, drug compounds, even DNA fragments. Subsequently, Chen et al. [22] have performed monolithic sol-gel column modified with L-hydroxyproline for chiral separation of danyl amino acids (Dns-AAs).

The application of gel monolithic column on microfluidic device integrates both the highly efficiency of monolithic column and the micromation of analytical instruments. Herr and Singh [23] had proposed a mode about cross-linked PAA gel for on-chip protein sizing. Proteins have been separated in $4 \mathrm{~mm}$ effective length of microchannel with efficiencies as high as $4.41 \times 10^{5}$ plates $/ \mathrm{m}$.

To explore a prompt and inexpensive way to separate the bio-molecules or chiral compounds, a novel PDMS microfluidic device with PAA gel monolithic column was prepared in an easy way. In this device, $\beta$-CD-bonded PAA gel was immobilized on the inner wall of PDMS-based microfluidic device through Bind-Silane. Allyl- $\beta-C D$ was used as crosslinker and chiral selector in gel matrix. The stability, transferring heat and optical characteristic of gel monolithic column on microchip are measured to tailor the feasibility of $\beta$-CD-bonded PAA gel monolithic column for hydrophobic PDMS microfluidic device.

\section{Experimental}

\subsection{Apparatus}

The analytes detection was performed by a home-built compactly laser-induced fluorescence (LIF) detector [24]. The entire detection system included a blue diode-pumped laser $(473 \mathrm{~nm})$ (Beijing Viasho Technology, China) which operated in constant mode at room temperature serving as excitation source, a photomultiplier tube (PMT) (Institute of Biophysics Chinese Academy of Sciences, China) for collecting fluorescence signal, a $530 \mathrm{~nm}$ interference filter and a holographic notch plus filter (476 nm) (Kaiser Optical System, Ann Arbor, MI, USA) above the PMT for filtering the excitation light. The laser was vertical to the separation microchannel by the leading of optical fiber; the PMT was upright to the separation microchannel and the optical fiber to avoid the disturbance from excitation light. The microfluidic device was driven by a high-voltage power supply (Shandong Chemical Engineering Institute, China).

\subsection{Chemicals}

Sylgard 184 PDMS polymer and curing agent were purchased from Dow Corning (Midland, MI, USA). Fluorescein isothiocyanate (FITC), Allylglycidyl ether (AGE), 3-(trimethoxysilyl)-propyl methacrylate (Bind-Silane), 2acrylamide-2-methylpropane-sulfonic acid (AMPS) and dansyl-threonine (Dns-Thr) were from Sigma (St. Louis, MO, USA). Acrylamide (AA) was bought from Tianjin Fucheng Chemical Regent Factory (Tianjin, China). $\beta$-Cyclodextrin (CD) was purchased from Shanghai Chemical Regents Company (Shanghai, China). N,N-Methylene-bisacrylamide (Bis) was from Tianjin Chemical Regents Institute (Tianjin, China). Ammonium persulfate (APS) was purchased from Tianjin Chemical Experimental Factory (Tianjin, China) and $N, N, N^{\prime}, N^{\prime}$-tetramethylethylenediamine (TEMED), urea, tris(hydroxymethyl)aminomethane (Tris) and boric acid were from Beijing Chemical Regents Limited Company (Beijing, China). L-Arginine (Arg), L-glutamine acid (Glu) and L-tryptophan (Trp) were purchased from Beijing Xinjingke Biotechnology Co. Ltd. (Beijing, China) L-cysteine (Cys) and L-phenylalanine (Phe) were from Nakarai Chemicals LTD (Kyoto, Japan). The above regents are all analytically pure.

\subsection{Device fabrication and sealing}

The process of fabrication PDMS-based microfluidic device includes fabricating template, casting PDMS replica, and sealing microchip.

Microfluidic channel pattern was designed by auto-CAD software, and corresponding PMMA master was fabricated by a method developed by our group [25]. Firstly, a glass negative mold was prepared using original micromachining technology including photolithographic pattern transformation and chemi- 
cal wet-etching. Then the PMMA positive replica-master was replicated from the glass negative mold with a thermal imprinting technique.

The PMMA replica-master was rinsed with deionized water and ethanol, and dried by nitrogen before casting. Sylgard 184 PDMS prepolymer and curing regent were mixed at 10:1 (w/w) ratio and degassed by vacuum $(0.1 \mathrm{MPa})$ for $1 \mathrm{~h}$. Subsequently, the mixture was cast against the PMMA replica-master and cured at $75^{\circ} \mathrm{C}$ for $2 \mathrm{~h}$. Then the PDMS replica containing channels was formed and peeled off from the PMMA replica-master. Four holes at the end of channels were chiseled for the reservoirs on PDMS replica.

The procedure of sealing PDMS microchip was described as: the PDMS replica containing channels and a PDMS slab were rinsed with ethanol and pure water orderly. They were located under a $30 \mathrm{~W}$ UV lamp (the wavelength is $254 \mathrm{~nm}$ ) for $1.5 \mathrm{~h}$ and then folded as soon as possible. It should be mentioned that the distance between the replica or slab and UV lamp is $5 \mathrm{~cm}$. At last, the folded chip was sandwiched between two glass clips for $24 \mathrm{~h}$ at least for firm sealing. The sealed PDMS-based microfluidic device was obtained and can be modified and filled gel in the next preparation of monolithic column. In our experiments, the microfluidic device we adopted is $50 \mu \mathrm{m}$ width and $17 \mu \mathrm{m}$ depth and its efficient separation length is $36 \mathrm{~mm}$.

\subsection{Preparation of PDMS-based microchip filled gel}

Firstly, the synthesis of allyl- $\beta-C D$ was performed in a wellventilated fume cupboard to avoid inhalation of the lacrymatory AGE referencing relative report [19], and the principle was shown in Fig. 1. The final solution was stored at $4{ }^{\circ} \mathrm{C}$ and used in experiment.

Sequentially, the monolithic column microfluidic device was prepared in the following process: the monomers containing $40 \mathrm{mg}$ acrylamide and $8.5 \mathrm{mg}$ AMPS and cross-link agent of $1.5 \mathrm{mg}$ Bis were dissolved in $0.30,0.50$ and $0.70 \mathrm{~mL}$ of $0.10 \mathrm{~mol} \mathrm{~L}^{-1}$ Tris $/ 0.15 \mathrm{~mol} \mathrm{~L}^{-1}$ boric acid buffer. Five hundred microliters of $0.1 \mathrm{~mol} \mathrm{~L}^{-1}$ allyl- $\beta-\mathrm{CD}$ (that means the concentration (w/v) of monomers are 5\%, 4\% and 3\%) was added. The monomers solution was degassed with nitrogen and 53, 67 or $100 \mu \mathrm{L}$ of $5 \%(\mathrm{w} / \mathrm{v})$ APS and 53,67 or $100 \mu \mathrm{L}$ of $5 \%(\mathrm{w} / \mathrm{v})$ TEMED were added and sucked immediately into Bind-Silanetreated channels by the syringe, which were pretreated with $0.4 \%$ (v/v) Bind-Silane solution containing $0.4 \%(\mathrm{v} / \mathrm{v})$ acetic acid for at least $2 \mathrm{~h}$. The polymerization started within a few seconds and completed within half an hour [20].

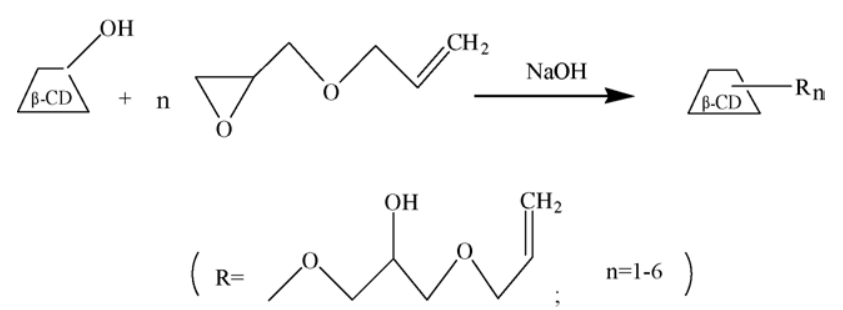

Fig. 1. The principle for preparation of allyl- $\beta-C D$.

\subsection{Separation of FITC-labeled AAs}

Before separation, Arg, Glu, Phe, Trp, Cys and Dns-Thr were labeled with FITC according to the procedure described in relative reference [23]. Additionally, the buffer containing $0.10 \mathrm{~mol} \mathrm{~L}^{-1}$ Tris- $-0.25 \mathrm{~mol} \mathrm{~L}^{-1}$ boric acid and $7 \mathrm{~mol} \mathrm{~L}^{-1}$ urea buffer ( $\mathrm{pH}$ 9.0) was acted running buffer for electrochromatography. In the injection process, a simple injection was adopted, and a potential of $800 \mathrm{~V}$ was applied between sample reservoir and sample waste reservoir for $30 \mathrm{~s}$. Subsequently, a voltage of $2500 \mathrm{~V}$ was supplied between buffer reservoir and buffer waste reservoir for the separation of FITC-labeled AAs in all experiments.

\section{Results and discussion}

\subsection{UV radiation and preparation of $P A A$ gel monolithcolumn}

Generally, plasma and UV mercury lamp are two irritation sources for sealing PDMS microfluidic device. Among them, UV mercury lamp was used customarily in ordinary chemistry laboratory because of its low price. In our works, UV mercury lamp (254 nm) was applied to seal the PDMS microfluidic device. Certainly, UV irradiation also generates active silanol groups in PDMS microchannel in the sealing of PDMS microfluidic device, which are important active groups to immobilize the PAA gel on the inner wall of PDMS microchannel.

Firstly, the silanol groups induced by UV irradiation can form firmly sealing through dehydration, and form $\mathrm{Si}-\mathrm{O}-\mathrm{Si}$ groups between two pieces of PDMS substrate. The principle can be interpreted as: UV irradiation on the surface of PDMS can accelerate the generation of silanol groups when the experiment was in air. Sandwiching the PDMS-based replica containing channel and PDMS slab between two glass clips can close up the two PDMS substrate, and was advantageous for the sealing [8]. Two days later, a firmly sealing of PDMS microfluidic device was accomplished.

Secondly, the silanol groups induced by UV irradiation are important active groups for immobilizing PAA gel monolithic column in PDMS microchannel. The silanol groups on inner wall of PDMS microchannel react with methoxy groups of BindSilane in acidic condition [26], which has a double bond and can join in the polymerization for preparation of PAA gel monolithic column. Therefore, Bind-Silane acted as a bifunctional molecule reacting with PDMS inner wall and polymerizing with monomers in preparation of PAA gel like an anchor.

\subsection{The characteristic of the $\beta$-CD-bonded PAA gel microchip}

As a medium used in the microfluidic device monolithic column electrochromatograph (EC), PAA gel is a classical stationary phase for separation of proteins, enzyme and AAs $[17,18]$. Its application and preparation in general gel chromatography is well developed. However some disadvantages of the PAA gel limits its application in CE: Firstly, the enduring potential of 
common PAA gel is about $300 \mathrm{~V} / \mathrm{cm}$, which is much lower for high efficient contemporary EC, and so seriously disturbs its widely application in EC. Secondly, the bubble generation in PAA gel for EC makes the separation in gel monolithic column difficult. Once the bubble formed in the gel monolithic column, the current would be broken down and the process of separation would be shut down compellingly. Therefore, much work has been done to improve the enduring potential and avoid the generation of bubble in PAA gel.

To enhance the enduring potential of PAA gel monolithic column, the PDMS-based microchannel was pretreated with acidic solution of Bind-Silane, which can combine the PAA gel and the wall of PDMS microchannel covalently to avoid formation of slots between the wall and the PAA gel. It also partly restrained the generation of bubble in gel monolithic column due to the infirm combination between the wall and the PAA gel. Therefore, the working potential of PAA gel was improved greatly to $800 \mathrm{~V} / \mathrm{cm}$ (it was applied in all separations), and its enduring potential is much higher than that of other reference reported [26].

Generally, the combination between hydrophilic PAA gel and hydrophobic PDMS is faint and unstable. PAA gel would be flushed out microchannel after frequently running without the solid chemical bond. In our experiments, the combination between PAA gel and PDMS microchannel is steady. It can be confirmed the PDMS-based microfluidic filling PAA gel remains highly efficiency for electrochromatography except accidental fracture.

In previous reports, bubble formation and leakage of gel are always the cumbersome puzzle and limit the application of gel media for highly efficient electrochromatography. In our works, the lifetime of microfluidic device PAA gel monolithic column was improved through the addition of allyl- $\beta-C D$. The stabil- ity of this microfluidic device was investigated by measuring the change of EOF within 15 days, and its alteration of EOF is about $1.18 \%$, which is much better than the original PDMS microfluidic device. Generally, The PDMS-based microfluidic device gel monolithic column remains highly separation efficiency for at least 1 month if it was store in water at $4{ }^{\circ} \mathrm{C}$. The further corroboration is that it remains good day-to-day repeatability for above 1 month: the RSD $(n=15)$ of retention time for the FITC in 15 days was $3.54 \%$.

On the other hand, gel is looked as an open-meshed lattice of long PAA chains which were tied at distant intervals by $\beta$ $\mathrm{CD}$ and Bis crosslinkers (see Fig. 2), whose structure is similar to the PAA gel. Based on the structure of PAA gel concerning the molecular-sieving effects, increasing the concentration of monomer in gel strands can decrease the pore size, and increase mechanical obstruction when the concentration of $\beta-C D$ was maintained in appropriate scope. To tailor monolithic column to the separation of FITC-labeled AAs, we designed a series of experiments to investigate the influence of the total concentration of monomers (the corresponding rates of each monomers are immovable) on the resolution characteristic of PAA (the concentration of $\beta-\mathrm{CD}$ was $25 \mathrm{mM}$ ). With the concentration of monomers decreased from $5 \%$ to $3 \%$ (w/v) (see Fig. 3), the migrating times of analytes became shorter and shorter. When the concentration of monomer is $4 \%$ as shown in Fig. 3 b, the particularity of PAA gel for separation is optimum, which remained the best resolution characteristic and appropriate migrating times for analytes.

\subsection{Separation of FITC-labeled amino acids}

To confirm the prompt separation ability of microfluidic device, five FITC-labeled Arg, Glu, Phe, Trp and Cys were sep-

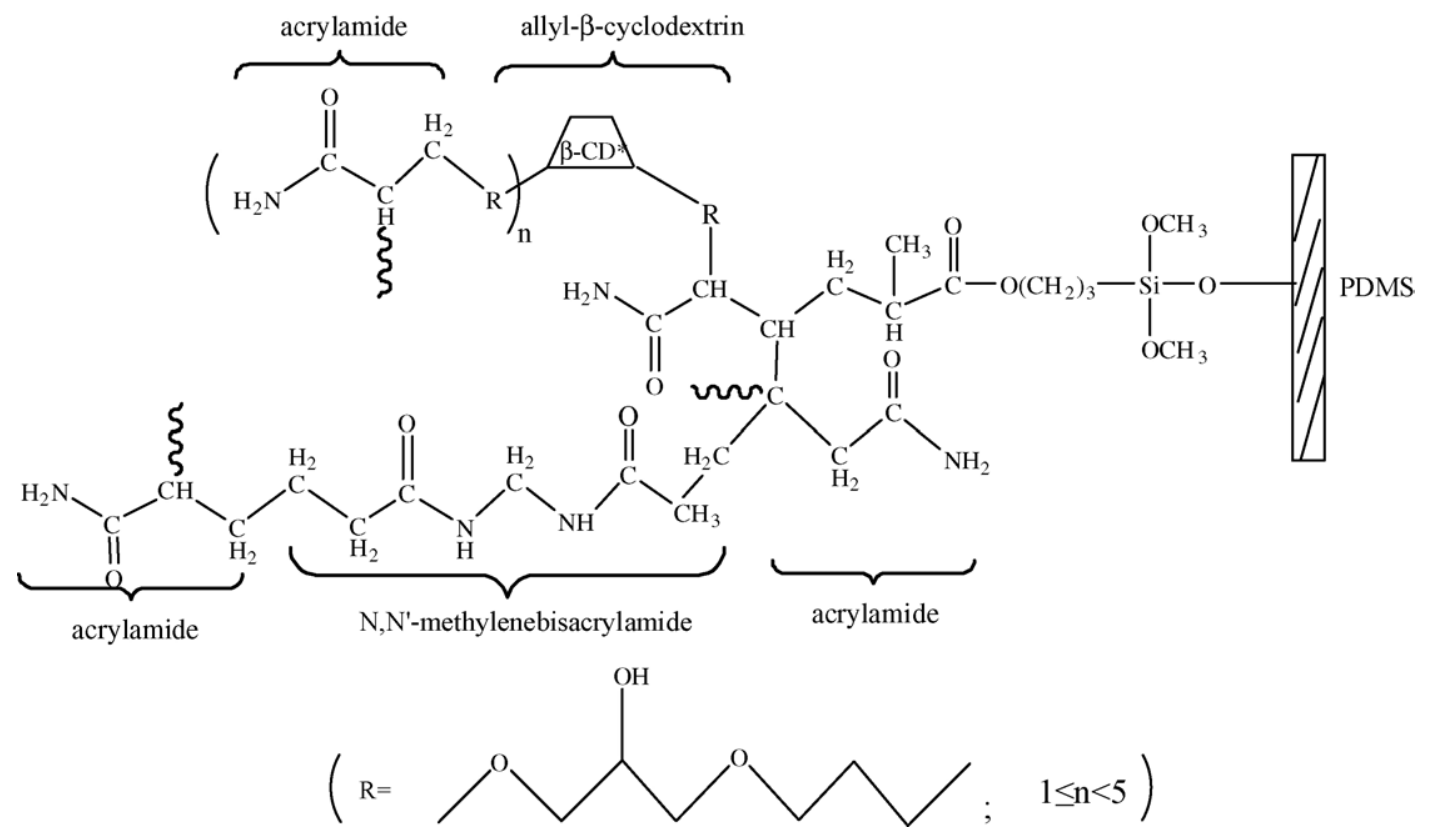

Fig. 2. The structure of $\beta-C D$ bonded PAA gel filling in PDMS microfluidic device. 

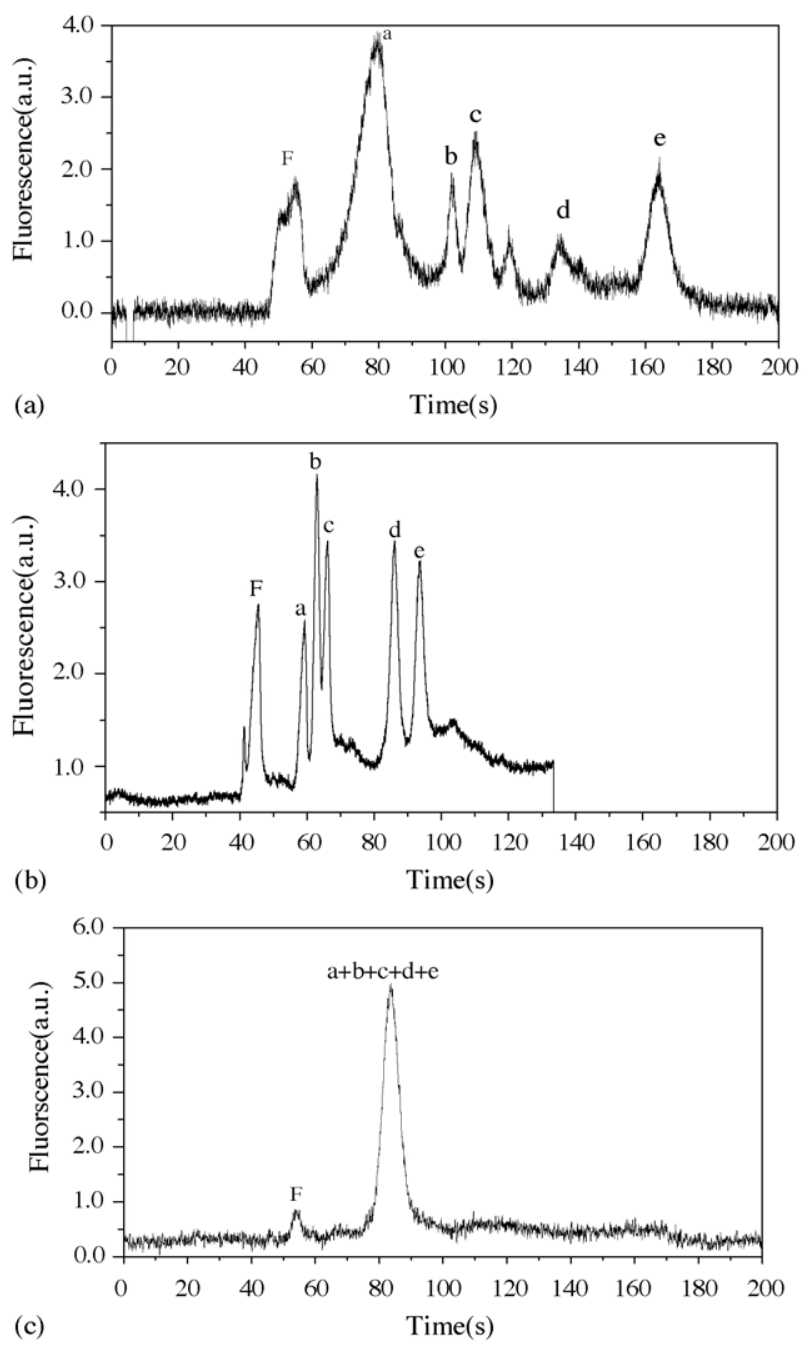

Fig. 3. The effect of concentration of monomers on the resolution of the FITClabeled Arg, Glu, Phe, Trp and Cys. The concentrations of allyl- $\beta-C D$ in three chromatograms are all $25 \mathrm{mM}$. The concentrations of monomers were: (A) $5 \%$; (B) $4 \%$; (C) $3 \%$. The separation potential is $2500 \mathrm{~V}$. The sample injecting potential is $800 \mathrm{~V}$ and the injection time is $60 \mathrm{~s}$. The running buffer is $0.10 \mathrm{~mol} \cdot \mathrm{L}^{-1}$ Tris $-0.25 \mathrm{~mol} \cdot \mathrm{L}^{-1}$ boric acid in $7.00 \mathrm{~mol} \mathrm{~L}^{-1}$ urea (pH 9.0). a, b, c, d, e and FITC are $5 \times 10^{-5} \mathrm{~mol} \mathrm{~L}^{-1}$ FITC-labeled Glu, Phe, Trp, Cys, Arg and FITC, respectively.

arated in the PDMS-based microfluidic device PAA gel monolithic column for EC. In our previous experiments [27], we have selected appropriate separation conditions including categories of buffer, the strength of voltage, $\mathrm{pH}$ value of running buffer and the modes of injection for separation. Pursuantly, five FITC-labeled Arg, Glu, Phe, Trp and Cys were separated in this microfluidic device. The chromatogram of separation was depicted in Fig. 3b. Generally, FITC-labeled AAs charged negative in alkaline buffer ( $\mathrm{pH} 9.0)$ move to positive electrode when the EOF of the gel media is small for amounts of AMPS. The migration times of AAs are different due to different properties of analytes, such as charge, size, molecular structure and mass. Several separation parameters were listed in Table 1. Five FITC-labeled AAs were successfully separated within $100 \mathrm{~s}$ and the maximum of theoretical plates can be reached to $2.06 \times 10^{5}$.
Table 1

The relevant parameters of the separation of $50 \mu \mathrm{M}$ FITC-labeling Glu, Phe, Trp, Cys, Arg and FITC

\begin{tabular}{lcccccc}
\hline Compound & Glu & FITC & Phe & Trp & Cys & Arg \\
\hline $\begin{array}{l}\text { Migration time (s) } \\
\text { Theoretical plates } \\
\left(\times 10^{5} \text { plates } / \mathrm{m}\right)\end{array}$ & 43 & 56 & 59 & 62 & 81 & 88 \\
\begin{tabular}{l} 
Resolution \\
\hline
\end{tabular} & 2.8 & 1.62 & 2.06 & 1.75 & 1.85 & 1.99 \\
\end{tabular}

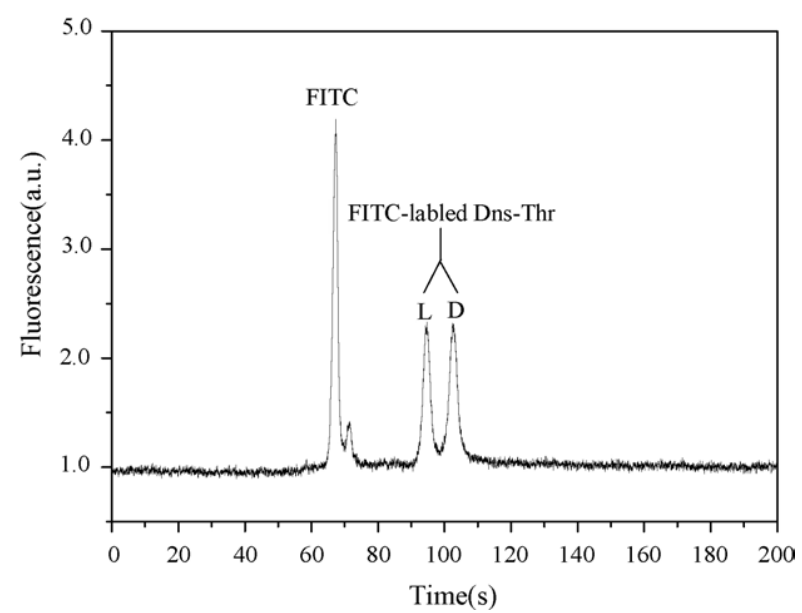

Fig. 4. The chromatogram of FITC-labeled Dns-Thr: the separation potential is $2000 \mathrm{~V}$. The injecting potential is $800 \mathrm{~V}$; the injection time is $30 \mathrm{~s}$; the running buffer is $0.10 \mathrm{~mol} \mathrm{~L}^{-1}$ Tris $-0.25 \mathrm{~mol} \mathrm{~L}^{-1}$ boric acid in $7.00 \mathrm{~mol} \mathrm{~L}^{-1}$ urea $(\mathrm{pH}$ 9.0).

Allyl- $\beta-C D$ as a chiral selector and crosslinker in PAA gel, it also exhibited efficient reorganization capability in PAA gel stationary phase. To test the chiral separation capability of $\beta$ CD bonded PAA monolithic column, a pair of FITC-labeled Dns-Thr was separated in a PDMS microchannel, the obtained chromatogram was shown in Fig. 4. It demonstrated the two optical isomers of Dns-Thr were separated absolutely in only $36 \mathrm{~mm}$ efficient separation length, and the resolution $\left(R_{\mathrm{S}}\right)$ of the enantiomers reached 1.63 .

\section{Conclusions}

In this paper, a novel fabricating technique for preparation PDMS-based microfluidic device gel monolithic column was proposed. The silanol group on inner wall of PDMS microchannel induced by the UV irradiation reacted with Bind-Silane, which was important anchor molecule was used to link gel. Therefore, the hydrophobicity of PDMS substrate was efficacious reformed by covalently bonding hydrophilic PAA gel, and the separation efficiency of PDMS microchannel was splendid improved. The results of experiments demonstrated the microfluidic device filling PAA gel has good stability. Moreover, it was found that the $\beta-C D$ bonded monolithic column possesses high efficiency for separation even for chiral separation. It would be a promising novel means for microfluidic device electrochromatography to prompt separation of large-molecule organic components. 


\section{Acknowledgments}

The authors gratefully acknowledge financial support of the National Science Fund for Distinguished Young Scholars of China (No. 20125514), National Natural Science Foundation of China (Nos. 20437020, 50273046) and Major Research Program of Chinese Academy of Sciences (KZCX3-SW-432).

\section{References}

[1] D.E. Raymond, A. Manz, H.M. Widmer, Anal. Chem. 66 (1994) 2858

[2] R. Su, J.-M. Lin, F. Qu, Z. Chen, Y. Guo, M. Yamada, Talanta 64 (2004) 1024.

[3] D.R. Reyes, D. Iossifidis, P.A. Auroux, A. Manz, Anal. Chem. 74 (2002) 2623.

[4] Z. Chen, Y. Gao, J.-M. Lin, R. Su, Y. Xie, J. Chromatogr. A 1038 (2004) 239.

[5] P.A. Auroux, D. Iossifidis, D.R. Reyes, A. Manz, Anal. Chem. 74 (2002) 2637.

[6] S.W. Hu, X.Q. Ren, M. Bachman, C.E. Sims, G.P. Li, N. Allbritton, Anal. Chem. 76 (2004) 1865

[7] A. Yamaguchi, P. Jin, H. Tsuchiyama, T. Masud, K. Sun, S. Matsuo, H. Misawa, Anal. Chim. Acta 468 (2002) 143.

[8] F. Meng, H. Chen, U. Fang, H. Zhu, Z. Fang, Chem. J. Chin. Univ. 23 (2002) 1264

[9] S. Lai, X. Cao, L.J. Lee, Anal. Chem. 76 (2004) 1175.

[10] S. Hu, X. Ren, M. Bachman, C.E. Sims, G.P. Li, N. Allbritton, Anal. Chem. 74 (2002) 4117.
[11] A.M. Spehar, S. Koster, V. Linder, S. Kulmala, N.F.R. Rooij, E. Verpoorte, H. Sigrist, W. Thormann, Electrophoresis 24 (2003) 3674.

[12] Y. Liu, J.C. Fanguy, J.M. Bledsoe, C.S. Henry, Anal. Chem. 72 (2000) 5939.

[13] S. Hu, X. Ren, M. Bachman, C.E. Sims, G.P. Li, N. Allbritton, Electrophoresis 24 (2003) 3679.

[14] B.E. Slentz, N.A. Penner, F.E. Regenier, J. Chromatogr. A 948 (2002) 225.

[15] M. Pumera, Talanta 66 (2005) 1048.

[16] S. Hiertén, Á. Vébvári, T. Srichaiyo, H.X. Zhang, J. Cap. Elec. 5 (1998) 13.

[17] M. Kato, H.M. Jin, K.K. Sakai, T. Toyo'oka, M.T. Dulay, R.N. Zare, J. Chromatogr. A 1004 (2003) 209.

[18] H.J. Tian, L.C. Brody, J.P. Landers, Genome Res. 10 (2000) 1403

[19] J.J. Coon, H.A. Steele, P.J. Laipis, W.W. Harrison, J. Proteome Res. 2 (2003) 610

[20] Á. Vébvári, A. Foldesi, C. Hetenyi, O. Kocnegarova, M.G. Schmid, V. Kudirkaite, S. Hjerten, Electrophoresis 21 (2000) 3116.

[21] T. Koide, K. Ueno, J. High Resol. Chromatogr. 23 (2000) 59.

[22] Z. Chen, T. Nishiyama, K. Uchiyama, T. Hobo, Anal. Chim. Acta 501 (2004) 17.

[23] A.E. Herr, A.K. Singh, Anal. Chem. 76 (2004) 4727.

[24] H. Li, J.-M. Lin, R. Su, K. Uchiyama, T. Hobo, Electrophoresis 25 (2004) 1907.

[25] H. Li, J.-M. Lin, R. Su, Z. Cai, K. Uchiyamo, Electrophoresis 26 (2005) 1825.

[26] S. Hierten, J. Chromatogr. 347 (1985) 191.

[27] J.-M. Lin, T. Nakagama, H. Okazawa, X.Z. Wu, T. Hobo, Fresenius J. Anal. Chem. 354 (1996) 451. 